\title{
Magnetic fluid hyperthermia induced by radiofrequency capacitive field for the treatment of transplanted subcutaneous tumors in rats
}

\author{
XU-HONG LI ${ }^{1}$, PENG-FEI RONG ${ }^{2}$, HE-KUN JIN ${ }^{3}$, WEI WANG ${ }^{2}$ and JIN-TIAN TANG ${ }^{4}$ \\ Departments of ${ }^{1}$ Rehabilitation, and ${ }^{2}$ Radiology, the Third Xiangya Hospital, \\ Central South University, Changsha, Hunan 410013; ${ }^{3}$ Department of Oncology, \\ Xiangya Hospital, Central South University, Changsha, Hunan 410008; \\ ${ }^{4}$ Institute of Engineering and Physics, Tsinghua University, Beijing 100084, P.R. China
}

Received August 26, 2011; Accepted November 22, 2011

DOI: $10.3892 /$ etm.2011.397

\begin{abstract}
Magnetic fluid hyperthermia (MFH) induced by a magnetic field has become a new heating technology for the treatment of malignant tumors due to its ability to heat the tumor tissue precisely and properly, and due to its significant therapeutic effects. In this study, MFH induced by radiofrequency capacitive field (RCF) for the treatment of transplanted subcutaneous tumors in rats, was investigated. A total of 50 rats bearing subcutaneous tumors were randomly divided into five groups, including i) a pseudo-treatment (PT) control group, ii) magnetic fluid (MF) group, iii) pure hyperthermia (PH) group, iv) magnetic fluid hyperthermia 1 (MFH1) group, and v) magnetic fluid hyperthermia 2 (MFH2) group. Tumors were irradiated for $30 \mathrm{~min}$ in the MFH1 group $24 \mathrm{~h}$ following injection of MF. Tumors were irradiated for $30 \mathrm{~min}$ in the MFH2 group $24 \mathrm{~h}$ following injection of MF, and irradiation was repeated for $30 \mathrm{~min} 72 \mathrm{~h}$ following injection of MF. Tumor volumes, tumor volume inhibition ratios and survival times in the rat model were examined. Temperatures of tumor cores and rims both rapidly reached the desired temperature $\left(\sim 50^{\circ} \mathrm{C}\right)$ for tumor treatment within 5 to $10 \mathrm{~min}$ in the MFH1 and MFH2 groups, and we maintained this temperature level by manually adjusting the output power (70-130 W). Tumor volumes of the MFH1 and MFH2 groups were reduced compared to those of the PT, MF and PH groups. The inhibitory effect on tumor growth in the MFH2 group (91.57\%) was higher compared to that in the MFH1 group (85.21\%) and the other groups. The survival time of the MFH2 group (51.62 \pm 2.28 days) and MFH1
\end{abstract}

Correspondence to: Professor Wei Wang, Department of Radiology, the Third Xiangya Hospital, Central South University, Changsha, Hunan 410013, P.R. China

E-mail:wawe01cn@yahoo.com.cn

Key words: magnetic fluid hyperthermia, radiofrequency capacitive field, transplanted subcutaneous tumor, rat group (43.10 \pm 1.57 days) was increased compared to that of the $\mathrm{PH}, \mathrm{MF}$ and PT groups. The results obtained show that MFH induced by RCF may serve as a potential and promising method for the treatment of tumors.

\section{Introduction}

High frequency hyperthermia is widely used in various countries as an adjuvant therapy for advanced tumors, including radiofrequency (RF) hyperthermia and microwave hyperthermia (1). Under such conditions, selective heating of the tumor is only possible when heat dissipation by blood flow in the normal tissue is much greater than that in the tumor tissues. Although both of these techniques are effective, currently RF is the preferred technique and the one most widely practiced, as $\mathrm{RF}$ is non-invasive and can produce localized deep heating (2). Yet RF hyperthermia techniques apply energy in an unfocused manner, and energy is delivered to both the tumor and normal tissues (3). The most serious shortcomings of RF hyperthermia in clinical use include no tissue-specific heating due to the indistinct border between the heating area and the non-heating area, over-heating in fat tissues and the requirement for a high output of power (1,000-2,000 W) (4).

In 1997 Jordan et al discovered that a nanoscaled magnetic fluid (MF) could be absorbed with much higher power in an alternating magnetic field, and used to treat diseases (5). This method is known as 'magnetic fluid hyperthermia' (MFH). Compared to other available hyperthermia modalities, MF, suspensions consisting of magnetic particles, is delivered to the tumor. An alternating magnetic field is then used to heat the particles and the corresponding tumor, thereby ablating it. In this way, focused heating of the particles is obtained in the regions where the static field is dominated by the alternating magnetic field (6-8).

In order to reduce the limitations of conventional RF thermotherapy and improve therapeutic anticancer activity, in this study the heating effects of magnetic nanoparticles were induced by radiofrequency capacitive field (RCF) with low power $(0-200 \mathrm{~W})$ and its treatment feasibility was investigated using Wistar rats bearing subcutaneous tumors. 


\section{Materials and methods}

Regents and instruments. $\mathrm{Fe}_{3} \mathrm{O}_{4} \mathrm{MF}$ was provided by the Institute of Medical Physics and Biomedical Engineering of Tsinghua University and characterized by a transmission electron microscope (TEM) (Hitachi H-600 instrument; Hitachi Corp., Tokyo, Japan). RCFs were produced by the Erbtherm1100 P hyperthermia system (27.12 MHz, 0-200 W, $11.0 \mathrm{~m}$ wavelength, Italy). RCF power was regulated by changing the output power of the system. Temperatures were measured by an IT-24P-Tiny thermocouple thermometer and 24 gauge polyurethane coated wire with polyester insulated thermocouple bead (Physitemp, US), and recorded dynamically by a temperature-recording instrument with 4 channels (Beijing Kunlun Tianchen Instrument Science and Technology Co., China).

Rats. Wistar rats (male, 4-5 weeks old) were purchased from the Institute of Dongchuan Animal Experimental Center, Central South University. The animal experiments were approved by the regional animal ethics committee and the rats were treated in accordance with the international animal ethics guidelines.

Walker-256 transplanted subcutaneous tumor models were established by implanting Walker-256 cells into the right thigh according to the previous literature (9).

When tumor diameters reached 0.8-1.0 cm 8-9 days following tumor implantation, 50 rats bearing subcutaneous tumors were randomly divided into five groups: i) the pseudotreatment (PT) control group, ii) MF group: injection of MF without hyperthermia, iii) pure hyperthermia $(\mathrm{PH})$ group: received one hyperthermia without injection of magnetic fluid, iv) magnetic fluid hyperthermia 1 (MFH1) group: received a single intratumoral injection of MF and one hyperthermia, v) magnetic fluid hyperthermia 2 (MFH2) group: received a single intratumoral injection of MF and two hyperthermias. Each group contained ten rats.

Hyperthermia test. MF was directly injected into the tumors at the 3,6,9 and 12 o'clock points with a volume equal to half of the tumor volume in the MF, MFH1 and MFH2 groups (10). The tumors were subjected to irradiation for $30 \mathrm{~min}$ in the MFH1 group $24 \mathrm{~h}$ following injection of MF. The tumors were subjected to irradiation for $30 \mathrm{~min}$ in the MFH2 group $24 \mathrm{~h}$ following injection of MF and irradiated repeatedly for $30 \mathrm{~min} 72 \mathrm{~h}$ following injection of MF. Similarly, tumors of the PH group underwent one hyperthermia. The Erbtherm1100 $\mathrm{P}$ hyperthermia system was applied to produce RCF, and the distance between the upper and lower electrodes placed on opposite sides of the right tumor region were $30 \mathrm{~mm}$. RCF parameters were carefully adjusted while the maximal temperature of rectal tissue was not above $40^{\circ} \mathrm{C}$ and the maximal temperature of the tumor core was maintained at $50^{\circ} \mathrm{C}$. Temperatures of different areas in rats were detected by IT-24P-Tiny thermocouple thermometers inserted into tumor cores, tumor rim, left leg tissue and rectal tissue of model rats and were recorded dynamically by a temperature-recording instrument with 4 channels.

Detection of thermotherapeutic effect. Computed tomography (CT) scanning was performed to document the intratumoral

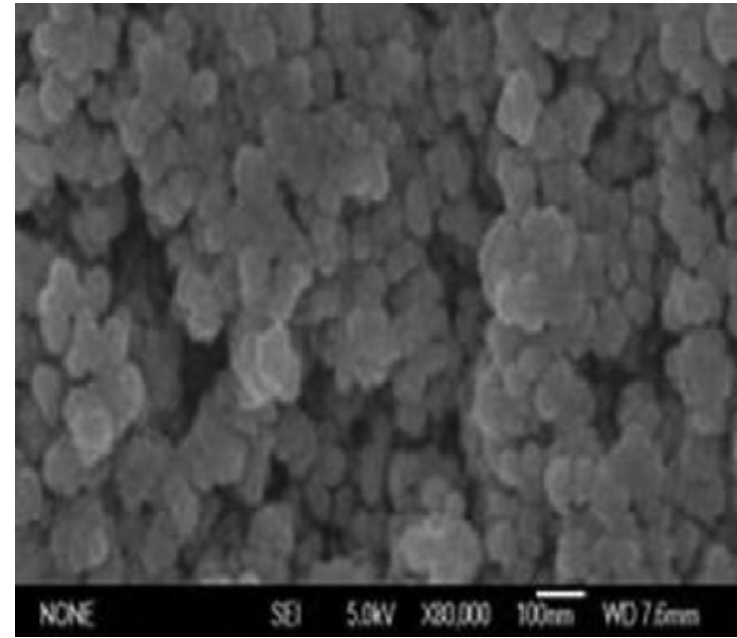

Figure 1. Transmission electron microscopy (TEM) images of $\mathrm{Fe}_{3} \mathrm{O}_{4}$ magnetic nanoparticles. The nanoparticles were approximately spherical, uniform in size, with an average diameter of approximately $10 \mathrm{~nm}$ (7 to $22 \mathrm{~nm}$ ) and had good dispersibility as evidenced by TEM.

distribution of magnetic nanoparticles one day after the first and second hyperthermia, respectively.

Tumor volumes were measured weekly according to the literature (11). Each tumor was measured with a sliding caliper to obtain a maximal diameter (a) and a minimal diameter (b), and tumor volume was calculated using the following formula: volume $=\mathrm{a} \times \mathrm{b} \times \mathrm{b} / 2$.

The tumor volume inhibition ratio was calculated as (1 - mean tumor volume of the experimental group/mean tumor volume of the control group) x $100 \%$. All rats were sacrificed for histopathological examination after six weeks. Rat survival time was examined according to the literature (12).

Statistical analysis. The Kaplan-Meier method was carried out to plot animal survival time. Values were expressed as the means \pm standard deviation (SD).

The data were analyzed by SPSS 13.0 statistical software. Heating rates were compared using the Chi-square $\left(\chi^{2}\right)$ test between the two groups. Differences in the results were considered to indicate statistical significance when $\mathrm{P}<0.05$.

\section{Results}

Characterization and detection of $M F$. The concentration of $\mathrm{Fe}_{3} \mathrm{O}_{4}$ in the $\mathrm{MF}$ was $32.93 \mathrm{mg} / \mathrm{ml}$. The TEM result indicates that magnetic particles were approximately spherical, approximately $10 \mathrm{~nm}$ diameter on average, round and had strong magnetism, as shown in Fig. 1.

Magnetic nanoparticles remained stable in the injected location without widespread delivery to other organs. A relative uniformity and homogeneity in intratumoral distribution of magnetic nanoparticles locally in targeted tumor tissues were demonstrated by CT and CT three-dimensional imaging (Fig. 2), so repeatable hyperthermias may be possible without repeatable injection of MF, and magnetic nanoparticles were not found in the other organs or tissues.

Diminished deposits of injected magnetic nanoparticles detected by $\mathrm{CT}$ imaging indicates that the local tumor under- 
A

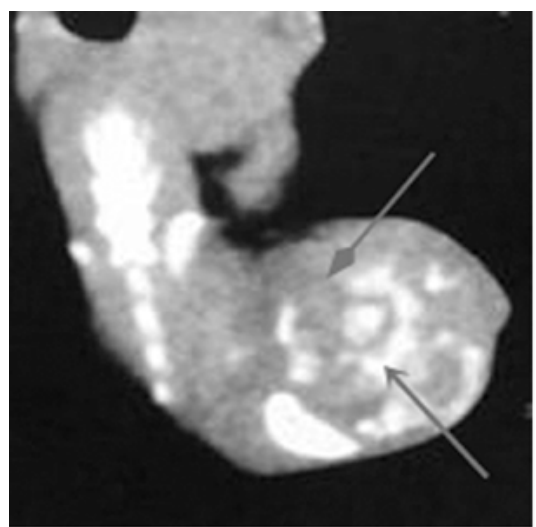

C

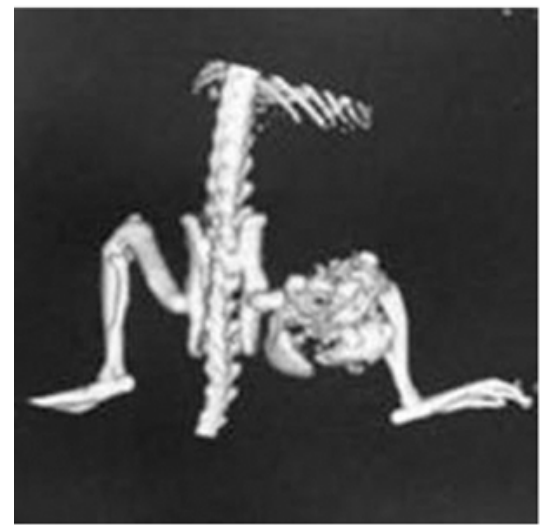

B

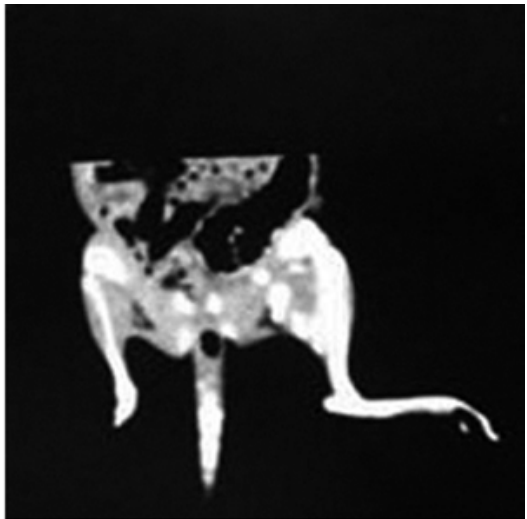

D

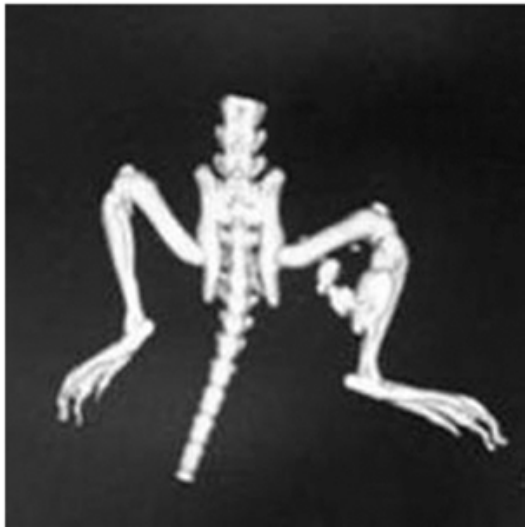

Figure 2. Spiral computed tomograph (CT) and three-dimensional images of subcutaneous tissue in the right thigh of rats at 1 day after (A and C) first hyperthermia and (B and D) second hyperthermia. MF had good dispersibility, with a high density [thick arrow, 346 hounsfield unit, (HU)], and a distinguishable CT attenuation number that was higher than that of tumor soft tissue (thin arrow, $52 \mathrm{HU}$ ). MF, magnetic fluid.
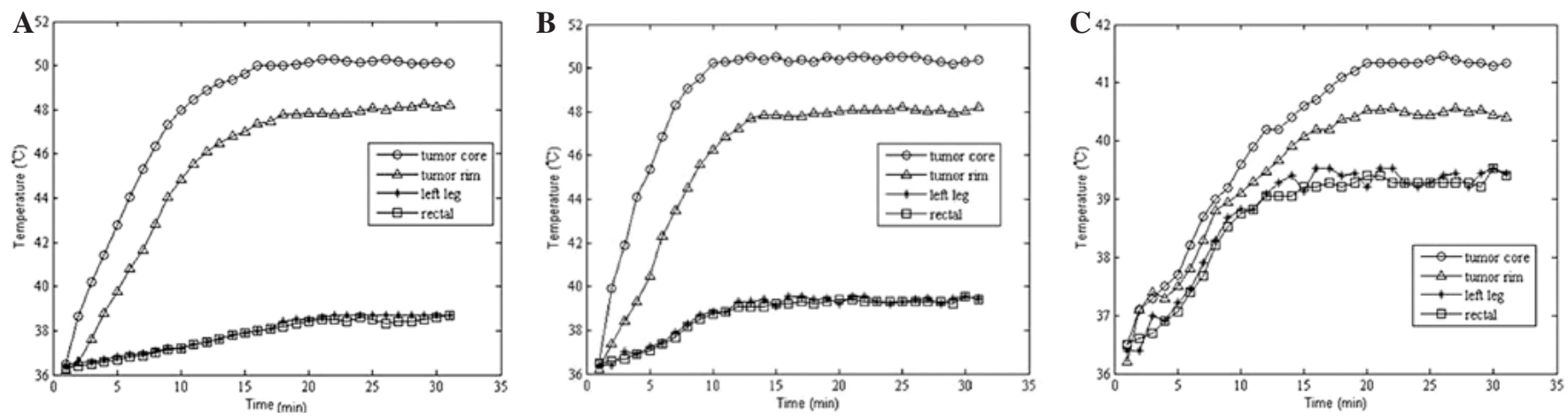

Figure 3. Time-dependent temperature protocols of tumor cores, tumor rims, left leg and rectal tissue in (A) the MFH1 group, (B) the MFH2 group and (C) the $\mathrm{PH}$ group. MFH, magnetic fluid hyperthermia; $\mathrm{PH}$, pure hyperthermia.

went necrosis or ulceration and broke away following the second hyperthermia treatment in the MFH2 group (Fig. 2). If further thermotherapy is required, magnetic nanoparticles could be injected repeatedly.

$M F H$ induced by RCF. Due to the intratumor injection of $\mathrm{Fe}_{3} \mathrm{O}_{4}$ magnetic nanoparticles and exposure to RCF, the temperatures of the tumor cores and rims rapidly reached the desired temperature $\left(\sim 50^{\circ} \mathrm{C}\right)$ for the treatment the tumor within 5 to 10 mins in the MFH1 and MFH2 groups, and were then maintained at a relatively constant level of 46 to $50^{\circ} \mathrm{C}$ by manually adjusting the output power $(70-130 \mathrm{~W})$. Temperatures of normal tissue in the left leg and rectum were raised slowly and were all below $40^{\circ} \mathrm{C}$ (Fig. 3). There was no statistical temperature difference between left leg tissue and rectal tissue $(\mathrm{P}=1.3)$; however, there was a statistical temperature difference between the other tissues $(\mathrm{P}=0.01)$. Whereas in the $\mathrm{PH}$ group, under the same RCF, temperatures of the four areas slowly reached $40^{\circ} \mathrm{C}$ within 15 to $20 \mathrm{~min}$, similarly, and then were maintained at a relatively constant level of $40^{\circ} \mathrm{C}$ by manually adjusting the output power to $70-150 \mathrm{~W}$ (Fig. 3). The tumor volume in the MFH1 and MFH2 groups decreased (Fig. 4).

Effects of MFH on tumor growth. Tumor growth in the MFH1 and MFH2 groups was inhibited and the boundaries 


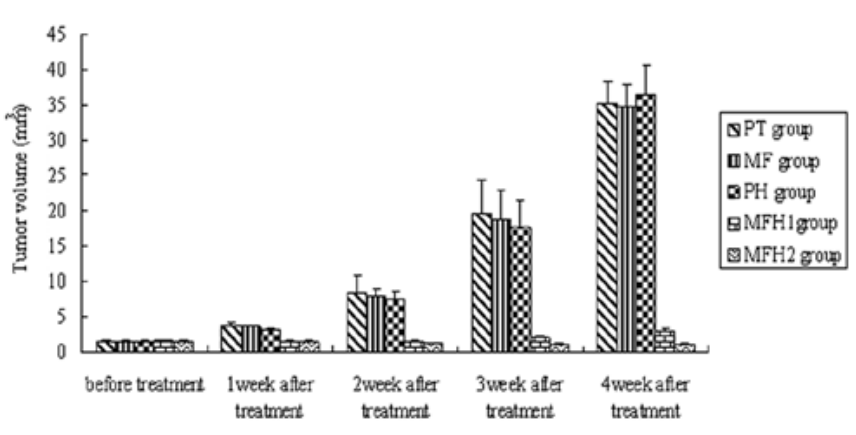

Figure 4. Changes in average tumor volume weekly following hyperthermia. PT, pseudo-treatment; MF, magnetic fluid; PH, pure hyperthermia; $\mathrm{MFH}$, magnetic fluid hyperthermia.

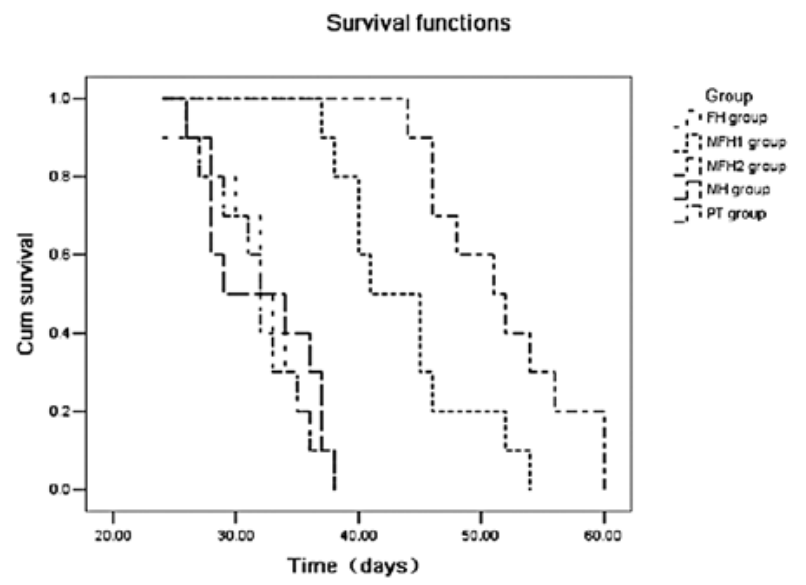

Figure 5. Kaplan-Meier survival curves of rats following treatment. PT, pseudo-treatment; MF, magnetic fluid; $\mathrm{PH}$, pure hyperthermia; MFH, magnetic fluid hyperthermia.

of coagulated areas were clear. Compared to the histopathological findings in these groups, thermocoagulation areas were unclear in the PT, MF and PH groups. In the MFH2 group, tumors of two model rats completely disappeared and there was no local recurrence for two months, and the tumor growth rates of the other eight model rats were markedly decreased. In the MFH1 group, the tumors of the model rats did not completely disappear. The tumor complete disappearance rate of the MFH2 group was $20 \%$ and showed more effective inhibition than the MFH1 group. Compared with the PT, MF and $\mathrm{PH}$ groups, tumor volumes of the MFH1 and MFH2 groups began to reduce from the first week after hyperthermia and the reduction was maintained until the fourth week after hyperthermia ( $\mathrm{P}=0.02$, Fig. 4). Tumor volume inhibition ratios in the MFH1 and MFH2 groups were 85.21 and $91.57 \%$, respectively, significantly higher than those observed in the $\mathrm{PH}, \mathrm{MF}$ and PT groups $(\mathrm{P}=0.01)$. There was no statistical difference in tumor volume among the $\mathrm{PT}, \mathrm{MF}$ and $\mathrm{PH}$ groups $(\mathrm{P}=1.2)$, and there was a statistical difference in tumor volume between the MFH1 and MFH2 group ( $\mathrm{P}=0.01)$.

Tumor volume inhibition ratios in the MFH1 group were 32.46 (first week), 50.62 (second week), 48.86 (third week) and $39.35 \%$ (fourth week) following thermotherapy; tumor volume inhibition ratios in the MFH2 group were 40.82 (first week), 72.32 (second week), 74.41 (third week) and
$73.26 \%$ (fourth week), respectively. Tumor inhibition in the $\mathrm{MFH} 2$ group was more effective than that of the MFH1 group.

Kaplan-Meier survival time analysis showed that the survival time of the MFH2 group (51.62 \pm 2.28 days) was longer than that of the MFH1 group (43.10 \pm 1.57 days), and also longer than that of the PH group (31.82 \pm 1.76 days), the MF group (32.50 \pm 1.85 days) and the PT group (32.15 \pm 1.25 days) (Fig. 5).

Pathological changes after thermotherapy. Histological examination revealed that a large amount of black iron was deposited in the tumor cells in the MF, MFH1 and MFH2 groups. The morphological features of tumor tissues in the MFH2 group were observed under a light-microscope $24 \mathrm{~h}$ after the two hyperthermia treatments, and included a detached or carbonated epidermis or degeneration and large patchy necrosis of tumor tissue. Entire tumor structures were completely destroyed and replaced by marked hemorrhage, large necrotic areas and red-dyed remnant without structure, or cavity, and some nuclei shrank, split and dissolved. MF deposition could be observed inside or outside of the necrosis region.

In the MFH 1 group, part of the tumor tissue became escharotic, and tumor volumes in the rats decreased following hyperthermia. On the second week after hyperthermia, the tumor began to grow and the tumor growth rate was slower than that of the PT, MF and PH groups. The degree of destroyed tumor tissue morphological structures in the MFH1 group $24 \mathrm{~h}$ after one hyperthermia treatment was lower than that in the MFH2 group. The tumor cell volume reduced and tumor tissue structure still survived. The coagulation necrosis area in the tumor tissue was smaller. Apoptotic changes, including contracted chromatin and deeply strained nucleus and apoptotic bodies, could be frequently found. Normal morphological tumor cells were distributed with a slice-shape in the border of the tumor tissue (Fig. 6).

Numerous black nanoparticles accumulated in the stroma of tumors, with widespread tumor necrosis surrounding the nanoparticles. Necrotic areas of the MFH2 group were larger than those of the MFH1 group (Fig. 6).

\section{Discussion}

Heat therapies such as hyperthermia and thermoablation are very promising approaches in the treatment of cancer. RCF hyperthermia is a modality that produces deep heating via conversion of electromagnetic energy to thermal energy. RF ablation with high frequency and high power (1,000-2,000 W) is a treatment for cancer that works by inserting a thin needle through the skin into a tumor guided by $\mathrm{CT}$ or ultrasound. Electrical energy is then delivered through a number of electrodes deployed through the needle, causing a zone of thermal destruction that encompasses the tumor (13).

RF ablation results in thermal injury as a consequence of friction that is generated by agitation of ions and is a commonly used technique for the treatment of localized tumors in the liver, with increasing application in other organs, such as the kidney, bone, lung, adrenal gland and prostate. Limitations of current RF ablation technology include the requirement for invasive needle placement, accuracy of image-guidance, tumor size limits, operator dependence and collateral damage to non-tumor tissue and adjacent structures (13). 
A

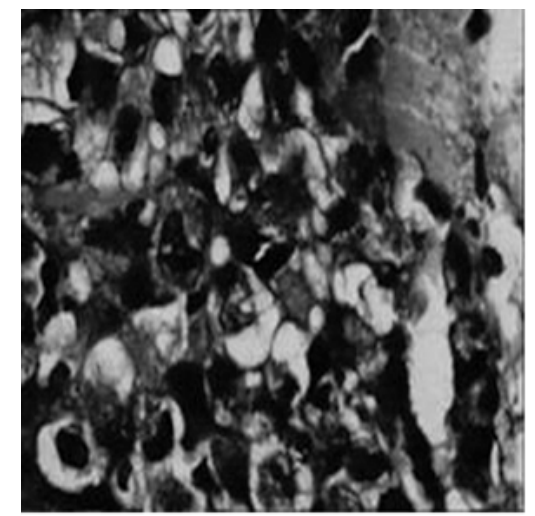

B

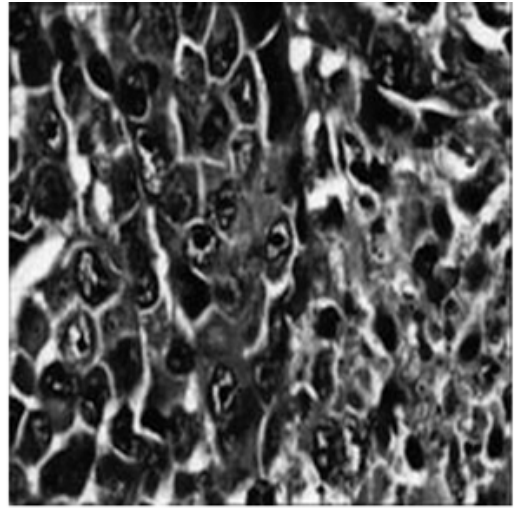

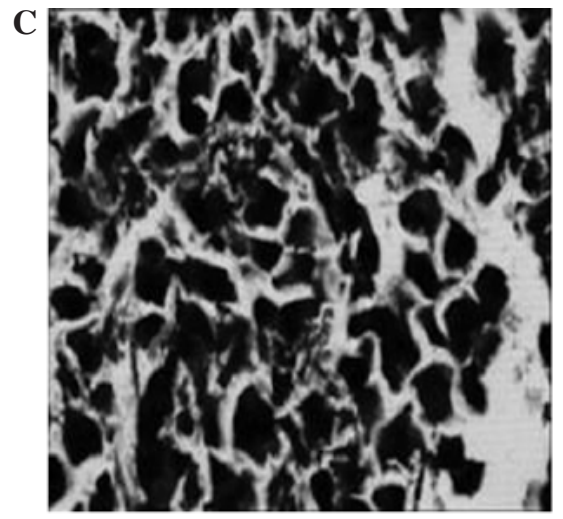

D

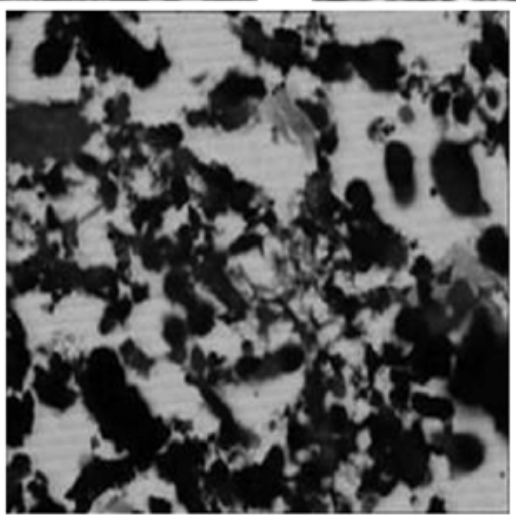

$\mathbf{E}$

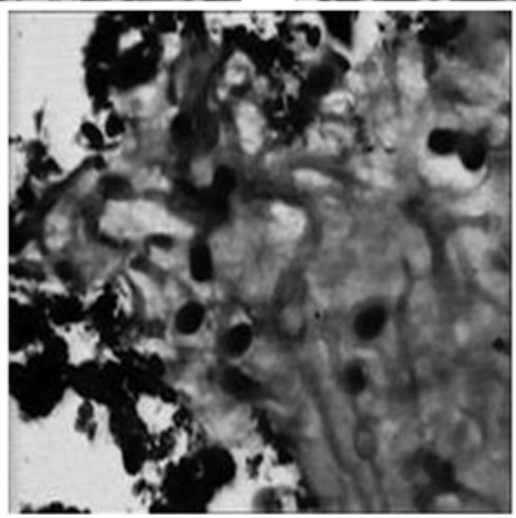

Figure 6. Morphological changes of tumor cell necrosis in (A) the pseudo-treatment group, (B) the magnetic fluid group, (C) the pure hyperthermia group, (D) the magnetic fluid hyperthermia 1 group and (E) the magnetic fluid hyperthermia 2 group under an optical microscope.

In non-invasive RCF hyperthermia, the major limiting factor is the inability of the electric field to focus on the tumor, so that all the tissues penetrated by the electric field are heated (14). RCF output power was significantly correlated with intra-tumor temperature, and it could be used as a parameter to assess efficacy of hyperthermia for the whole tumor region.

In general, malignant cells are more sensitive to heat in the range $41-45^{\circ} \mathrm{C}$ than normal cells. In addition, the majority of clinically apparent tumors have blood perfusion rates less than $20 \%$ of those of the surrounding normal tissue, meaning that they may be preferentially heated. The minimum temperature for therapeutic benefit was above $42^{\circ} \mathrm{C}$. Above $46^{\circ} \mathrm{C}$ the time for cell killing becomes quite short and the different sensitivity of malignant and benign cells disappears; above $50^{\circ} \mathrm{C}$ all cells are killed very quickly (15). In the capacitive heating technique, the current spread can also cause excessive surface heating by the output of high power, so it is impossible for a therapeutic temperature of $46-50^{\circ} \mathrm{C}$ to be used. In our study, the temperature of the tumor was not above $42^{\circ} \mathrm{C}$ in the FH group, and there was no significant difference in therapeutic benefit in the FH group, compared with the PT and MF groups.

Routine medical use of RF is $13.56 \mathrm{MHz}$ ( $22 \mathrm{~m}$ wavelength), 27.12 MHz (11 m wavelength) and $40.68 \mathrm{MHz}(7.5 \mathrm{~m}$ wavelength). The frequency of $27.12 \mathrm{MHz}$ is most commonly used in the non-invasive method, as the use of higher frequencies results in a decreased depth of penetration. RCF with 27.12 MHz and 0-200 W RF electromagnetic waves as a source of heat produces deep heating via conversion of electromagnetic energy to thermal energy. Oscillation of high-frequency electrical and magnetic fields produces movement of ions, rotation of polar molecules and distortion of non-polar molecules, with resultant heat generation. However, the heating effect of MF under RCF with $27.12 \mathrm{MHz}$ and $0-200 \mathrm{~W}$ for treating tumors has been not reported $(16,17)$.

In this study, the desired temperature $\left(50^{\circ} \mathrm{C}\right)$ of $\mathrm{MF}$ induced by non-invasive RCF for cancer hyperthermia was obtained, and the temperature of the target tumor area with magnetic particles rapidly reached $50^{\circ} \mathrm{C}$ within $5 \mathrm{~min}$ at a power of $150 \mathrm{~W}$ and the tumor could undergo necrosis. Compared to surrounding tissue, limited higher density imaging of MF deposits in the tumor was observed clearly by CT scanning (Fig. 2). Compared with the PT, MF and PH groups, large areas of necrosis and a marked inhibitory effect on tumor growth were found in the MFH1 and MFH2 groups, which indicates that MFH had a significant therapeutic effect on tumors in model rats, and thermotherapy in the MFH2 group was the best therapeutic agent among all the groups tested. In contrast to conventional hyperthermic techniques, hyperthermia using magnetic nanoparticles under RCF with only a low power of $70-150 \mathrm{~W}$ enhanced the temperature to reach the target of $50^{\circ} \mathrm{C}$ without any substantial damage to the surrounding tissue. The three-dimensional thermal analysis could be developed in further studies.

The results obtained by CT showed that there was no evidence of injury to other organs in rats by using magnetic nanoparticles. Nanosized $\mathrm{Fe}_{3} \mathrm{O}_{4}$ magnetic nanoparticles are a new kind of biomaterial without cytotoxic effects (Fig. 2).

The use of MF provides an impetus for developing a non-invasive manner for RCF. MFH promises to be a viable alternative in the treatment of localized cancerous tumors. 
MFH for tumor therapy could improve regional control and decrease the risk of complications, as it has good power absorption capabilities in a high-frequency alternating electromagnetic field.

In conclusion, following injection of MF in rat tumors and exposure to RCF with a low power $(150 \mathrm{~W})$, the tumor core and tumor rim can both rapidly reach the desired temperature $\left(\sim 50^{\circ} \mathrm{C}\right)$ within 5 to 10 mins and maintain a relatively stable-state intratumoral temperature for tumor treatment. Tumor volumes of the MFH1 and MFH2 group were smaller than those of the PT, MF and PH groups. MFH induced by RCF shows a marked inhibitory effect on tumor growth in model rats and may be a potential and promising method with better heat localization and focusing abilities for treating tumors.

\section{Acknowledgements}

The authors thank Lin-Yun Zhao, Shao-Wen Wang and Xiao-Dong Zhang of the Institute of Engineering and Physics at Tsinghua University for valuable discussion on the preparation of magnetic fluid suspensions. This study was supported by Projects 30571779 and 10775085 of the National Natural Science Foundation of China and project (2009SK3171) of the Hunan Provincial Science and Technology Department.

\section{References}

1. Habash RW, Bansal R, Krewski D and Alhafid HT: Thermal therapy, part 1: an introduction to thermal therapy. Crit Rev Biomed Engl 34: 459-489, 2006.

2. Stauffer PR: Evolving technology for thermal therapy of cancer. Int J Hyperthermia 21: 731-744, 2005.

3. Hildebrandt B, Rau B, Gellermann J, et al: Standards and perspectives in locoregional hyperthermia. Wien Med Wochenschr 154: 148-158, 2004.

4. Hager ED, Dziambor H, Höhmann D, Gallenbeck D, Stephan M and Popa C: Deep hyperthermia with radiofrequencies in patients with liver metastases from colorectal cancer. Anticancer Res 19: 3403-3408, 1999.
5. Jordan A, Scholz R, Wust P, et al: Effects of magnetic fluid hyperthermia (MFH) on $\mathrm{C} 3 \mathrm{H}$ mammary carcinoma in vivo. Int $\mathrm{J}$ Hyperthermia 13: 587-605, 1997.

6. Latorre M and Rinaldi C: Applications of magnetic nanoparticles in medicine: magnetic fluid hyperthermia. P R Health Sci J 28 227-238, 2009

7. Cherukuri P, Glazer ES and Curley SA: Targeted hyperthermia using metal nanoparticles. Adv Drug Deliv Rev 62: 339-345, 2010.

8. Shenoi MM, Shah NB, Griffin RJ, Vercellotti GM and Bischof JC: Nanoparticle preconditioning for enhanced thermal therapies in cancer. Nanomedicine 6: 545-563, 2011.

9. Luo X, Hu L, Sun C, Xiong L and Mi Y: An experimental study of energy controllable steep pulse in the treatment of rat with subcutaneous transplantive tumor. Sheng Wu Yi Xue Gong Cheng Xue Za Zhi 24: 492-495, 2007.

10. Cheng FY, Su CH, Yang YS, et al: Characterization of aqueous dispersions of $\mathrm{Fe}(3) \mathrm{O}(4)$ nanoparticles and their biomedical applications. Biomaterials 26: 729-738, 2005.

11. Jordan A, Scholz R, Gneveckow U, et al: Description and characterization of the novel hyperthermia and thermoablation-system MFH $300 \mathrm{~F}$ for clinical magnetic fluid hyperthermia. Med Phys 10: 1444-1451, 2004.

12. Camargo CA, da Silva ME, da Silva RA, Justo GZ, Gomes-Marcondes MC and Aoyama H: Inhibition of tumor growth by quercetin with increase of survival and prevention of cachexia in Walker 256 tumor-bearing rats. Biochem Biophys Res Commun 406: 638-642, 2011.

13. Flanders VL and Gervais DA: Ablation of liver metastases: current status. J Vasc Interv Radiol 21: S214-222, 2010.

14. Glazer ES and Curley SA: Radiofrequency field-induced thermal cytotoxicity in cancer cells treated with fluorescent nanoparticles. Cancer 116: 3285-3293, 2010.

15. Cardinal J, Klune JR, Chory E, Jeyabalan G, Kanzius JS, Nalesnik M and Geller DA: Noninvasive radiofrequency ablation of cancer targeted by gold nanoparticles. Surgery 144: 125-132, 2008.

16. Banerjee R, Katsenovich Y, Lagos L, McIintosh M, Zhang X and Li CZ: Nanomedicine: magnetic nanoparticles and their biomedical applications. Curr Med Chem 17: 3120-3141, 2010.

17. Johannsen M, Gneveckow U, Taymoorian K, et al: Thermal therapy of prostate cancer using magnetic nanoparticles. Actas Urol Esp 31: 660-667, 2007. 